\title{
Molecular alterations contributing to pancreatic cancer chemoresistance.
}

Rajabpour $A^{1}$, Rajaei $F^{2}$, Teimoori-Toolabi L ${ }^{3}$.

\section{Author information}

1Cellular and Molecular Research Center, Qazvin University of Medical Sciences, Qazvin, Iran; Department of Molecular Medicine, School of Medicine, Qazvin University of Medical Sciences, Qazvin, Iran; Department of Molecular Medicine, Pasteur Institute of Iran, Tehran, Iran.

2Cellular and Molecular Research Center, Qazvin University of Medical Sciences, Qazvin, Iran; Department of Molecular Medicine, School of Medicine, Qazvin University of Medical Sciences, Qazvin, Iran.

3Department of Molecular Medicine, Pasteur Institute of Iran, Tehran, Iran. Electronic address: Iteimoori@pasteur.ac.ir.

\begin{abstract}
Pancreatic ductal adenocarcinoma (PDAC) is one of the most common causes of cancer-related death all over the world. This disease is difficult to treat and patients have an overall 5-year survival rate of less than 5\%. Although two drugs, gemcitabine (GEM) and 5-fluorouracil (5-FU) have been shown to improve the survival rate of patients systematically, they do not increase general survival to a clinically acceptable degree. Lack of ideal clinical response of pancreatic cancer patients to chemotherapy is likely to be due to intrinsic and acquired chemoresistance of tumor cells. Various mechanisms of drug resistance have been investigated in pancreatic cancer, including genetic and epigenetic changes in particular genes or signaling pathways. In addition, evidence suggests that microRNAs (miRNAs) play significant roles as key regulators of gene expression in many cellular processes, including drug resistance. Understanding underlying genes and mechanisms of drug resistance in pancreatic cancer is critical to develop new effective treatments for this deadly disease. This review illustrates the genes and miRNAs involved in resistance to gemcitabine in pancreatic cancer.
\end{abstract}

Copyright @ 2016 IAP and EPC. Published by Elsevier B.V. All rights reserved.

\section{KEYWORDS:}

Drug resistance; Gemcitabine; Genes; MicroRNAs; Pancreatic ductal adenocancinoma 